\title{
Complicación en recambio de prótesis tumoral no convencional
}

\author{
Complication in replacement of unconventional tumor prosthesis
}

\author{
Jiménez-García LM, ${ }^{*}$ Chávez-Valenzuela A, ${ }^{\ddagger}$ Caballero-López CG,${ }^{\S}$ López-Ortiz DB ${ }^{\natural}$
}

Hospital Universitario de Puebla, BUAP.

RESUMEN. Las metástasis óseas de un cáncer primario de cualquier parte del cuerpo son la forma más común de tumor óseo maligno constituyendo aproximadamente $70 \%$ de ellos. Siendo el fémur proximal la localización extravertebral más común para su localización; $10 \%$ de los pacientes presentan alguna fractura patológica, lo que es una grave complicación. En la actualidad, los objetivos del tratamiento de una fractura patológica son: resección con márgenes oncológicos adecuados, que el paciente sobreviva la intervención quirúrgica, mantener la funcionalidad, que el implante colocado tenga un tiempo de vida más largo que el del paciente. El uso de prótesis de cadera no convencionales es una excelente opción de tratamiento cumpliendo los cuatro objetivos establecidos, por ello se presenta este caso en el que se realiza una hemiartroplastía de cadera derecha bipolar con una prótesis no convencional donde se cursa con una complicación y se realiza la cirugía de resolución.

Palabras clave: Tumor, fractura, cadera, complicación, hueso.

\section{Introducción}

Las fracturas de cadera en general representan un importante gasto en la salud pública a nivel internacional, se estima que en los Estados Unidos de América se producen cerca de 280,000 fracturas de cadera al año, se espera que se duplique para el 2050. Cerca de nueve de 10 fracturas de este tipo
ABSTRACT. Bone metastases from a primary cancer of any part of the body are the most common form of malignant bone tumor constituting approximately $70 \%$ of them. The proximal femur being the most common extravertebral location for its location; $10 \%$ of patients have some pathological fracture, which is a serious complication. Currently the objectives of the treatment of a pathological fracture are: resection with adequate oncological margins, that the patient survives the surgical intervention, maintain the functionality, that the placed implant has a longer life time than the patient. The use of non-conventional hip prostheses is an excellent treatment option fulfilling the 4 established objectives, therefore this case is presented in which a right bipolar hip hemiarthroplasty is performed with a non-conventional prosthesis where it was treated with a described complication and the surgery of resolution.

Keywords: Tumor, fracture, hip, complication, bone. se producen en pacientes mayores a los 65 años, siendo más frecuentes en pacientes femeninos que en masculinos en una relación 3:1; otros factores de riesgo para este tipo de fracturas son raza caucásica, déficit neurológico, malnutrición, problemas visuales, tumores malignos y escasa actividad física. ${ }^{1}$

El cáncer es la segunda causa de mortalidad en adultos con cerca de 565,650 muertes. Las metástasis óseas son

\footnotetext{
* Residente de tercer año de Traumatología y Ortopedia.

${ }^{\ddagger}$ Residente de cuarto año de Traumatología y Ortopedia.

${ }^{\S}$ Asesor metodológico, Alergólogo Pediatra.

ๆ Jefe del Servicio de Traumatología y Ortopedia del Hospital Universitario de Puebla BUAP.
}

Correspondencia:

Luis Manuel Jímenez-García

Villa de las Gardenias 4-B, manzana 44, Villas de Atlixco 2, Cholula, Puebla, México, Tel: 331-5173-620.

E-mail: luisjimenez0522@gmail.com

Recibido: 12-06-2019. Aceptado: 30-06-2021.

Citar como: Jiménez-García LM, Chávez-Valenzuela A, Caballero-López CG, López-Ortiz DB. Complicación en recambio de prótesis tumoral no convencional. Acta Ortop Mex. 2021; 35(3): 286-289. https://dx.doi.org/10.35366/102369 
causa común de morbilidad en pacientes con tumores malignos, cuyas consecuencias pueden ser dolor refractario a la mayoría de los analgésicos convencionales, desórdenes metabólicos, fracturas patológicas y afección espinal que puede producir una compresión severa. ${ }^{2}$ Las metástasis óseas de un cáncer primario de cualquier parte del cuerpo son la forma más común de tumor óseo maligno, constituyendo aproximadamente $70 \%$ de ellos; ${ }^{2,3}$ asimismo son reportadas como la primera manifestación de malignidad en 5 a $20 \%$ de los pacientes con cáncer de origen desconocido.

El esqueleto, después de los pulmones y el hígado, es el tercer sitio más frecuente de la enfermedad ósea metastásica, ${ }^{2,4,5}$ siendo el fémur proximal la localización extravertebral más común para su localización; $10 \%$ de los pacientes presentan alguna fractura patológica, lo que es una grave complicación, ya que deteriora la calidad de vida por el aumento del dolor y la incapacidad funcional que éstas producen, incrementando incluso el riesgo de muerte en $20 \%$.

Los objetivos del tratamiento quirúrgico de la lesión son cuatro: 1) resección con márgenes oncológicos adecuados; 2) que el paciente sobreviva a la intervención quirúrgica; 3) mantener la funcionalidad; y 4) que el implante colocado tenga un tiempo de vida más largo que el del paciente. ${ }^{4,5,6} \mathrm{En}$ 1940, Austin Moore y Harold Bohlman realizaron el primer reemplazo femoral proximal para un tumor óseo. El primer reporte de prótesis femoral total metálica fue realizado por Buchman en 1965 y en los años 80 se desarrollaron los sistemas protésicos modulares. ${ }^{6}$

La región por debajo del trocánter menor es el límite anatómico para la aplicación de un sistema convencional versus un sistema no convencional. ${ }^{7}$ Las indicaciones son: lesiones neoplásicas, pérdidas traumáticas, infecciones, osteólisis masiva por enfermedad del cemento, prótesis convencionales con fractura por debajo del vástago, etcétera.

Las prótesis no convencionales comparten características como diseño individual. En la prótesis RIMAG ${ }^{\circledR}$ existe la posibilidad de dos vástagos: V1 tiene el propósito de re-

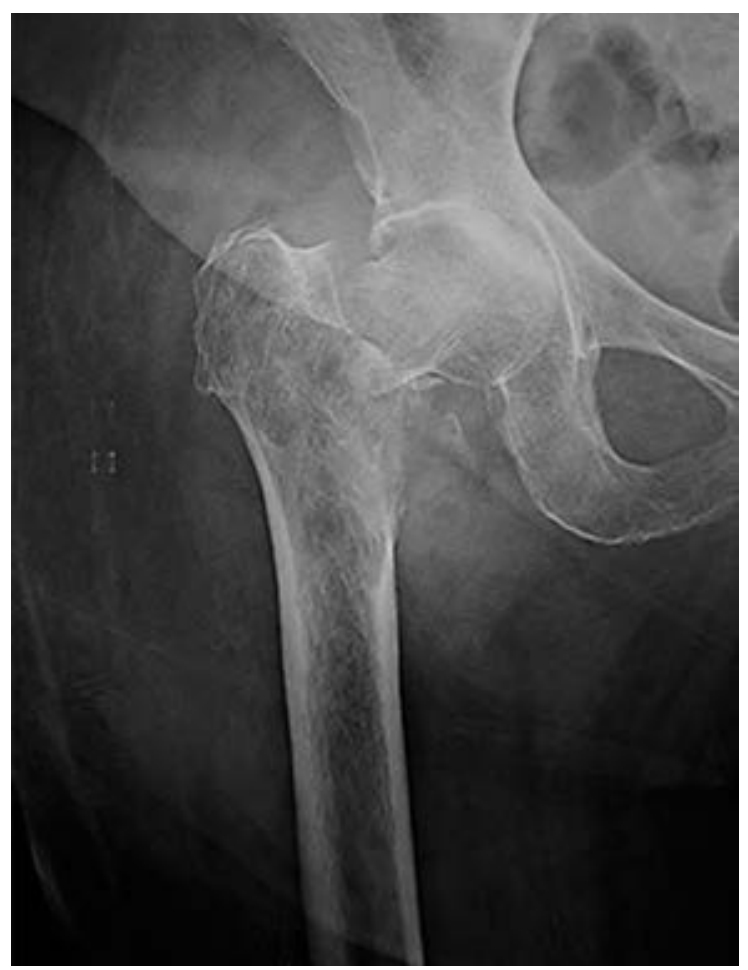

Figura 1: Radiografía anteroposterior de cadera derecha con solución de continuidad transcervical, adecuada congruencia articular coxofemoral.
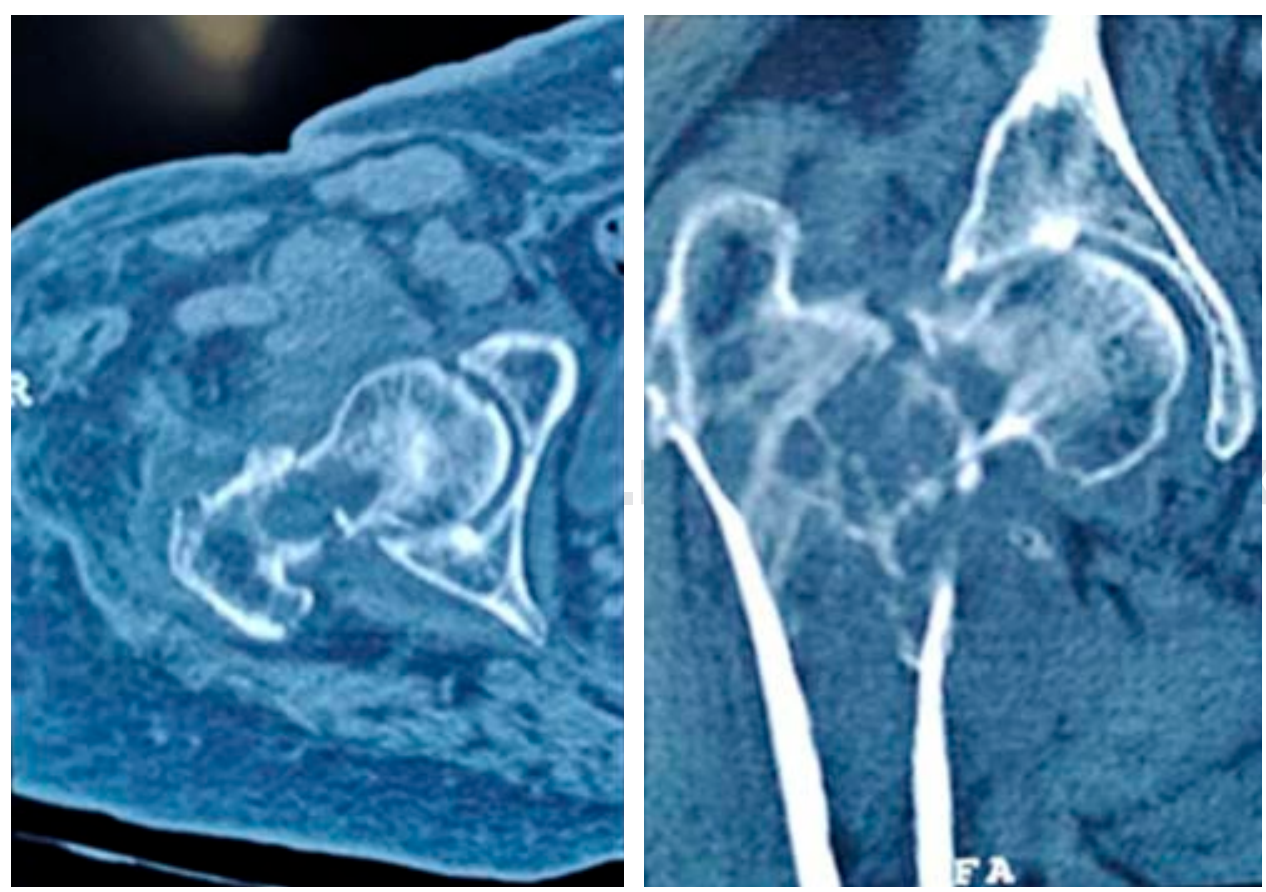

Figura 2:

Tomografía simple de cadera derecha donde se observan múltiples lesiones líticas en sitio de fractura. 

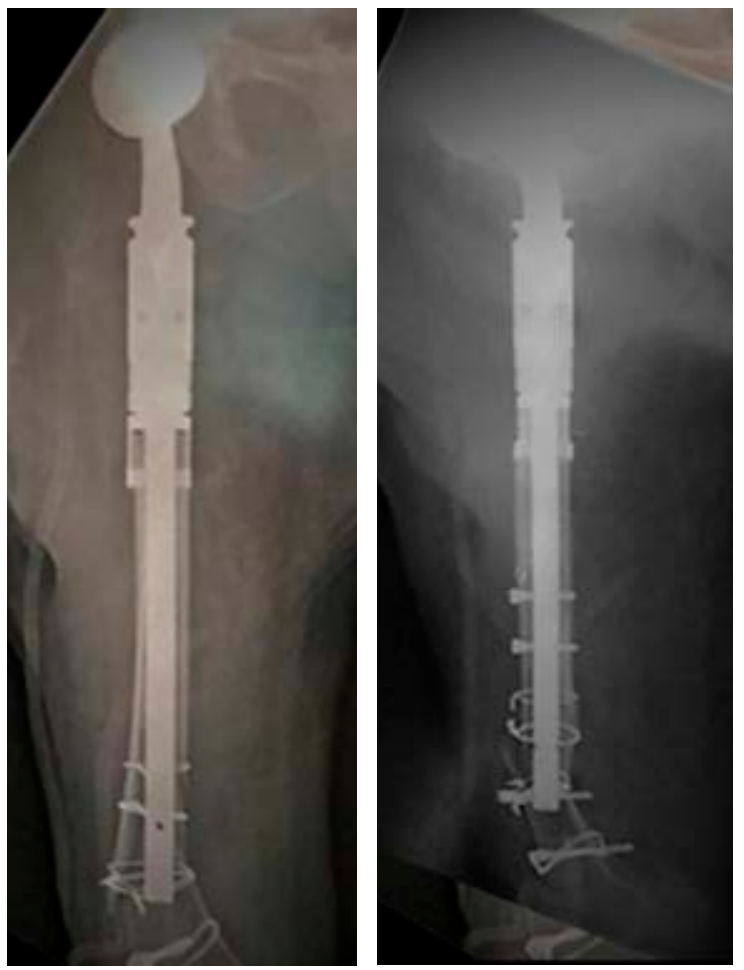

Figura 3: Radiografía postquirúrgica. Anteroposterior de cadera con complicación transquirúrgica tipo fractura periprotésica por torsión Vancouver tipo 2 y con osteosíntesis insuficiente.

construir el área vecina al trocánter menor o calcar femoral diseñado para reconstruir sólo los primeros $11 \mathrm{~cm}$ proximales del fémur y el V2 tiene la finalidad de reconstruir el fémur a partir de los $12 \mathrm{~cm}$ distales a la cabeza femoral, un objetivo importante es evitarse brazos de palanca que puedan inducir fracturas.

\section{Presentación de caso}

Mujer de 66 años con antecedentes de diabetes mellitus tipo 2, fractura cerrada de tobillo derecho de cuatro años de evolución con tratamiento quirúrgico, adecuada evolución, fascitis necrotizante posterior a laparatomía exploradora con tratamiento y resolución quirúrgica, tabaquismo de 50 años a razón de tres diarios. Inicia su padecimiento actual al intentar pararse del inodoro, escucha chasquido y crujido en cadera derecha, que se acompañó de dolor intenso 10/10 de EVA, incapacidad funcional y caída de plano de sustentación. A la exploración física extremidad pélvica derecha con hipotrofia de cuádriceps, sin aumento de volumen, equimosis o deformidad, dolor a la palpación, arcos de movimiento y fuerza no valorable por dolor, acortamiento de $1 \mathrm{~cm}$, actitud de rotación externa. Se realiza radiografía simple (Figura 1) y tomografía simple de cadera derecha (Figura 2), donde se observa solución de continuidad trazo simple oblicuo transcervical, asimismo se observan múltiples lesiones líticas en sitio de fractura, por lo que previo a interconsulta a servicios oncológicos se solicita gammagrafía ósea, la cual descarta proceso infeccioso, por lo que con estudios de extensión refieren enfermedad ósea metastásica avanzada con primario desconocido. Decidimos tratamiento con hemiartroplastía bipolar con prótesis no convencional para cadera (RIMAG ${ }^{\circledR}$ R2-V2), con resección $20 \mathrm{~cm}$ de fémur proximal, tuvimos como complicación transquirúrgica una fractura periprotésica (Figura 3) por torsión en diáfisis femoral distal supracondílea Vancouver tipo 2, se intentó corregir sin éxito colocando cerclajes. Por lo que se programó a un segundo turno quirúrgico para reducción abierta y fijación interna con colocación de placa anatómica LC-DCP de fémur distal derecho y cable quirúrgico colocándose en defecto óseo distal injerto óseo heterólogo, también se realiza cambio de espaciador y tornillo de extensión de prótesis tumoral no convencional, con adecuada reducción postquirúrgica y recuperación de longitud y orientación femoral (Figura 4). Muestra patológica de fémur proximal reporta metástasis de adenocarcinoma moderadamente diferenciado con patrón papilar en zona de cuello e intertrocantérica que condicionó fractura con bordes y límites quirúrgicos libres de neoplasia.

\section{Discusión}

En la actualidad, es controvertido el tratamiento definitivo de las fracturas de cadera en terreno patológico, ya que
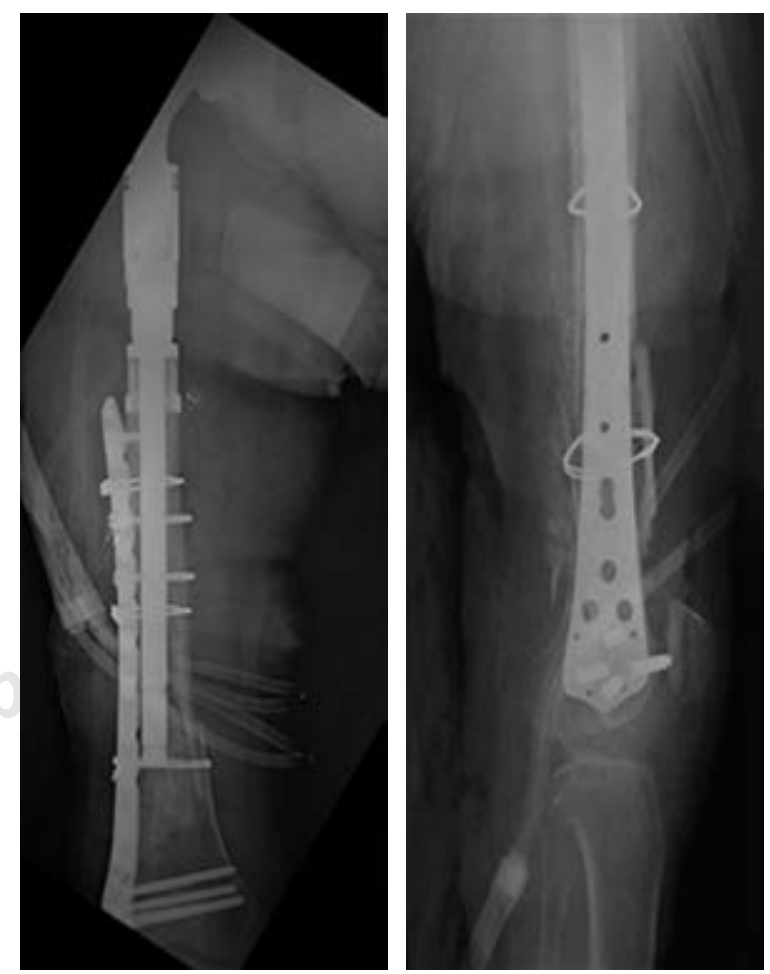

Figura 4: Radiografía postoperatoria anteroposterior y lateral de fémur derecho colocación de placa anatómica LC-DCP de fémur distal derecho y cable quirúrgico colocándose en defecto óseo distal injerto óseo heterólogo. 
en la literatura se habla de artroplastía u osteosíntesis, con sus limitantes y beneficios. Sin embargo, debido a que la osteosíntesis presenta un tiempo estimado de 4.5 meses en generar consolidación ${ }^{4}$ y la no remisión de dolor postquirúrgica, se ha optado por la artroplastía en tiempos recientes debido a la posibilidad de cumplimiento de los tres objetivos para tratamiento de pacientes de este tipo. ${ }^{4,5}$ La implantación de una prótesis total de tipo tumoral con resección ósea de toda la lesión metastásica se reserva en el caso de personas con buen estado general, con esperanza de vida mayor o igual a un año, con la región acetabular sana.

Las principales complicaciones de la artroplastía son: recurrencia local, infección, aflojamiento aséptico, falla mecánica y fractura (prótesis o hueso), ${ }^{5}$ por lo que pese a la simplificación de la colocación de la prótesis, las complicaciones son un factor importante a tomar en cuenta, ya que, aunque en bajo porcentaje, son capaces de cambiar el pronóstico mecano-funcional y de supervivencia del paciente y, por lo general, la resolución de las mismas no es sencilla, ya que influyen diferentes factores específicos. En términos de supervivencia de la persona, es muy difícil estimar el papel de tratamiento quirúrgico o la elección del implante, algunos autores reportan $12 \%$ de decesos durante el primer mes postquirúrgico, ${ }^{5}$ de igual manera para optar por un tratamiento de este tipo se debe tener en cuenta el factor económico, ya que comparado con la osteosíntesis, es mucho más elevado el costo.

Existen pocos estudios para el tratamiento de las complicaciones de la colocación de prótesis no convencionales de cadera. Se debe tener en cuenta el tipo de caso y el estado general del mismo para la elección de tratamiento definitivo, ya sea un implante tipo artroplastía u osteosíntesis. Los pacientes con fractura en terreno patológico deben ser indi- vidualizados, bien estudiados y con un amplio conocimiento del sistema a utilizar para reducir al máximo las posibles complicaciones a generarse.

Referencias

1. LaVelle DG. Campbell cirugía ortopédica; fracturas y luxaciones. Madrid, España: Marbán; Libro 6; Capítulo 52, 2013, 2804-6.

2. Sánchez-Torres LJ, Ruiz-Tenorio A, Chávez-Reyna MM, RodríguezDomínguez EA, Rascón-Álvarez O, Santos-Hernández M. Origen de la enfermedad ósea metastática quirúrgica. Acta Ortop Mex. [Internet]. Disponible en: http://www.medigraphic.com/pdfs/ortope/or-2013/ or133i.pdf

3. Greenspan A, Remagen W. Tumores de huesos y articulaciones. Madrid: Marbán; Capítulo 8, 2002, 367-88.

4. Doung YC, Kenan S, Rapp T. Metastatic lesions of the proximal femur. Bull NYU Hosp Jt Dis. 2011; 69(1): 81-6. Disponible en: https://pdfs. semanticscholar.org/1c74/51208d437459f9daf43d8b0a04fd7bab43ea.pdf

5. Trujillo-González R, Nieto-Lucio L, Mendoza-Breton JA. Incidencia de fracturas patológicas por metástasis en el fémur proximal en un hospital de concentración. Acta Ortop Mex. [Internet]. Disponible en: http://www.medigraphic.com/pdfs/ortope/or-2016/or164d.pdf

6. Rico-Martínez G, Delgado-Cedillo EA, Linares-González LM, Lindoro-Félix H, León-Hernández SR. Eficacia de la prótesis no convencional para la cadera tipo RIMAG en pacientes con tumores óseos primarios, metástasis y pérdidas osteoarticulares no tumorales. Acta Ortop Mex. [Internet]. 2013; 27(5): 299-304. Disponible en: http://www.medigraphic.com/pdfs/ortope/or-2013/or135d.pdf

7. Rico MG, Domínguez HV, Muller JA, Delgado CEA, Miranda RJA, Montoya CR. Diseño de una prótesis no convencional bloqueada para cadera (RIMAG) a partir de mediciones de fémures mexicanos. Acta Ortop Mex. [Internet]. 2008; 22(2): 70-9. Disponible en: http://www. medigraphic.com/pdfs/ortope/or-2008/or082b.pdf

Conflicto de intereses: Los autores y los centros de investigación con los que están afiliados no han recibido pagos financieros u otros beneficios de cualquier entidad comercial relacionada con el tema de este artículo. 